\title{
Pi, Euler Numbers, and Asymptotic Expansions
}

\section{J. M. Borwein, P. B. BorweIn, and K. DilCher ${ }^{1}$, Dalhousie University, Halifax, Canada}

JONATHAN M. BoRwein was an Ontario Rhodes Scholar (1971) at Jesus College, Oxford, where he completed a D. Phil. (1974) with Michael Dempster. Since 1974 he has worked at Dalhousie University where he is professor of mathematics. He has also been on faculty at Carnegie-Mellon University (1980-82). He was the 1987 Coxeter-James lecturer of the Canadian Mathematical Society and was awarded the Atlantic Provinces Council on the Sciences 1988 Gold Medal for Research. His research interests include functional analysis, classical analysis, and optimization theory.

Peter B. Borwein obtained a Ph.D. (1979) from the University of British Columbia, under the supervision of David Boyd. He spent 1979-80 as a NATO research fellow in Oxford. Since then he has been on faculty at Dalhousie (except for a sabbatical year at the University of Toronto) and is now Associate Professor of Mathematics. His research interests include approximation theory, classical analysis, and complexity theory.

KARL DILCHER received his undergraduate education and a Dipl. Math. degree at Technische Universität Clausthal in West Germany. He completed his Ph.D (1983) at Queen's University in Kingston, Ontario with Paulo Ribenboim. Since 1984 he has been teaching at Dalhousie, where he is now Assistant Professor. His research interests include Bernoulli numbers and polynomials, and classical complex analysis.
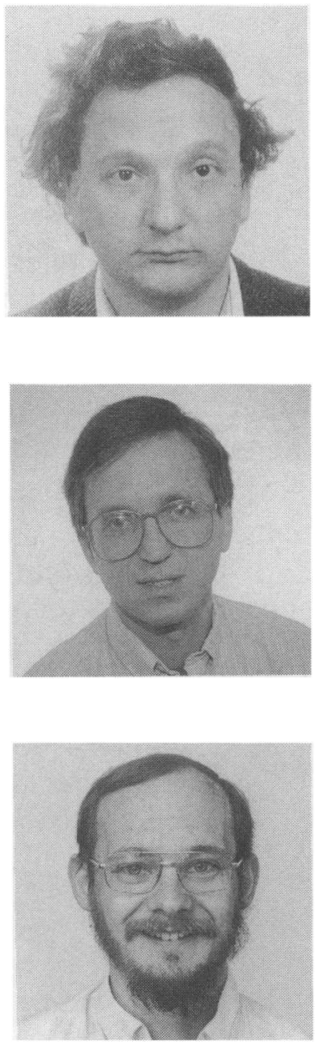

1. Introduction. Gregory's series for $\pi$, truncated at 500,000 terms, gives to forty places

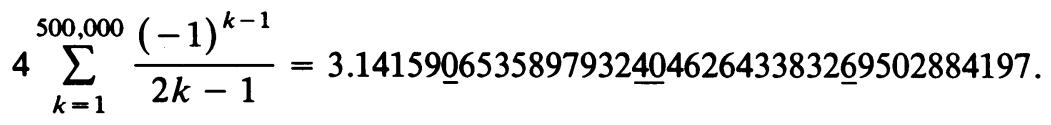

The number on the right is not $\pi$ to forty places. As one would expect, the 6th digit after the decimal point is wrong. The surprise is that the next 10 digits are correct. In fact, only the 4 underlined digits aren't correct. This intriguing observation was sent to us by R. D. North [10] of Colorado Springs with a request for an explanation. The point of this article is to provide that explanation. Two related

\footnotetext{
${ }^{1}$ Research of the authors supported in part by NSERC of Canada.
} 
examples, to fifty digits, are

$$
\begin{aligned}
& \frac{\pi}{2} \doteq 2 \sum_{k=1}^{50,000} \frac{(-1)^{k-1}}{2 k-1} \\
& =1.5707 \underline{8} 632679489 \underline{1} 619231321 \underline{1916397520520985833147388} \\
& \begin{array}{llll}
1 & -1 & 5 & -61
\end{array}
\end{aligned}
$$

and

$$
\begin{aligned}
& \log 2 \doteq \sum_{k=1}^{50,000} \frac{(-1)^{k+1}}{k} \\
& =.6931 \underline{371806} 69945309397232121474176568048300134 \underline{46572} \text {, } \\
& \begin{array}{lllll}
1 & -1 & 2 & -16 & 272
\end{array}
\end{aligned}
$$

where all but the underlined digits are correct. The numbers under the underlined digits are the numbers that must be added to correct these. The numbers $1,-1,5$, -61 are the first four Euler numbers while 1, - 1, 2, -16, 272 are the first five tangent numbers. Our process of discovery consisted of generating these sequences and then identifying them with the aid of Sloane's Handbook of Integer Sequences [11]. What one is observing, in each case, is an asymptotic expansion of the error in Euler summation. The amusing detail is that the coefficients of the expansion are integers. All of this is explained by Theorem 1 .

The standard facts we need about the Euler numbers $\left\{E_{i}\right\}$, the tangent numbers $\left\{T_{i}\right\}$, and the Bernoulli numbers $\left\{B_{i}\right\}$, may all be found in [1] or in [6]. The numbers are defined as the coefficients of the power series

$$
\begin{aligned}
\sec z & =\sum_{n=0}^{\infty}(-1)^{n} \frac{E_{2 n} z^{2 n}}{(2 n) !} \\
\tan z & =\sum_{n=0}^{\infty}(-1)^{n+1} \frac{T_{2 n+1} z^{2 n+1}}{(2 n+1) !} \quad \text { and } T_{0}=1 \\
\frac{z}{e^{z}-1} & =\sum_{n=0}^{\infty} \frac{B_{n} z^{n}}{n !} .
\end{aligned}
$$

They satisfy the relations

$$
\begin{gathered}
\sum_{k=0}^{n}\left(\begin{array}{c}
2 n \\
2 k
\end{array}\right) E_{2 k}=0, \quad E_{2 n+1}=0, \\
B_{n}=\frac{-n T_{n-1}}{2^{n}\left(2^{n}-1\right)} \quad n \geqslant 1,
\end{gathered}
$$

and

$$
\sum_{k=0}^{n}\left(\begin{array}{c}
n+1 \\
k
\end{array}\right) B_{k}=0
$$

These three identities allow for the easy generation of $\left\{E_{n}\right\},\left\{T_{n}\right\}$, and $\left\{B_{n}\right\}$. The 
first few values are recorded below.

\begin{tabular}{|c|c|c|c|c|c|c|c|c|c|}
\hline$n$ & 0 & 1 & 2 & 3 & 4 & 5 & 6 & 7 & 8 \\
\hline$E_{n}$ & 1 & 0 & -1 & 0 & 5 & 0 & -61 & 0 & 1365 \\
\hline$T_{n}$ & 1 & -1 & 0 & 2 & 0 & -16 & 0 & 272 & 0 \\
\hline$B_{n}$ & 1 & $\frac{-1}{2}$ & $\frac{1}{6}$ & 0 & $\frac{-1}{30}$ & 0 & $\frac{1}{42}$ & 0 & $\frac{-1}{30}$ \\
\hline
\end{tabular}

It is clear from (1.4) that the Euler numbers are integral. From (1.5) and (1.6) it follows that the tangent numbers are integers. Also,

$$
\left|E_{2 n}\right| \sim \frac{4^{n+1}(2 n) !}{\pi^{2 n+1}} \text { and }\left|B_{2 n}\right| \sim \frac{2(2 n) !}{(2 \pi)^{2 n}}
$$

as follows from (5.1) and (5.2) below. The main content of this note is the following theorem. The simple proof we offer relies on the Boole Summation Formula, which is a pretty but less well-known analogue of Euler summation. The details are contained in Sections 2 and 3 (except for c] which is a straightforward application of Euler summation). More complicated developments can be based directly on Euler summation or on results in [9].

THEOREM 1. The following asymptotic expansions hold:

$$
\begin{aligned}
\frac{\pi}{2}-2 \sum_{k=1}^{N / 2} \frac{(-1)^{k-1}}{2 k-1} & \sim \sum_{m=0}^{\infty} \frac{E_{2 m}}{N^{2 m+1}} \\
& =\frac{1}{N}-\frac{1}{N^{3}}+\frac{5}{N^{5}}-\frac{61}{N^{7}}+\cdots \\
\text { b] } \log 2-\sum_{k=1}^{N / 2} \frac{(-1)^{k-1}}{k} & \sim \frac{1}{N}+\sum_{m=1}^{\infty} \frac{T_{2 m-1}}{N^{2 m}} \\
& =\frac{1}{N}-\frac{1}{N^{2}}+\frac{2}{N^{4}}-\frac{16}{N^{6}}+\frac{272}{N^{8}}-\cdots
\end{aligned}
$$

and

c]

$$
\begin{aligned}
\frac{\pi^{2}}{6}-\sum_{k=1}^{N-1} \frac{1}{k^{2}} & \sim \frac{1}{2 N^{2}}+\sum_{m=0}^{\infty} \frac{B_{2 m}}{N^{2 m+1}} \\
& =\frac{1}{N}+\frac{1}{2 N^{2}}+\frac{1}{6 N^{3}}-\frac{1}{30 N^{5}}+\frac{1}{42 N^{7}} \cdots .
\end{aligned}
$$

From the asymptotics of $\left\{E_{b}\right\}$ and $\left\{B_{n}\right\}$ and (1.5) we see that each of the above infinite series is everywhere divergent; the correct interpretation of their asymptotics is

$$
\begin{array}{ll}
\left.\mathrm{a}^{\prime}\right] & \sum_{m=1}^{\infty} \frac{E_{2 m}}{N^{2 m}}=\sum_{m=1}^{K} \frac{E_{2 m}}{N^{2 m}}+0\left(\frac{(2 K+1) !}{(\pi N)^{2 K+1}}\right) \\
\left.\mathrm{b}^{\prime}\right] & \sum_{m=1}^{\infty} \frac{T_{2 m-1}}{N^{2 m}}=\sum_{m=1}^{K} \frac{T_{2 m-1}}{N^{2 m}}+0\left(\frac{(2 K+1) !}{(\pi N)^{2 K-1}}\right) \\
\left.\mathrm{c}^{\prime}\right] & \sum_{m=1}^{\infty} \frac{B_{2 m}}{N^{2 m+1}}=\sum_{m=1}^{K} \frac{B_{2 m}}{N^{2 m+1}}+0\left(\frac{(2 K+1) !}{(2 \pi N)^{2 K+1}}\right),
\end{array}
$$


where in each case the constant concealed by the order symbol is independent of $N$ and $K$. In fact, the constant 10 works in all cases.

2. The Boole Summation Formula. The Euler polynomials $E_{n}(x)$ can be defined by the generating function

$$
\frac{2 e^{t x}}{e^{t}+1}=\sum_{n=0}^{\infty} E_{n}(x) \frac{t^{n}}{n !} \quad(|t|<\pi)
$$

(see [1, p. 804]). Each $E_{n}(x)$ is a polynomial of degree $n$ with leading coefficient 1. We also define the periodic Euler function $\bar{E}_{n}(x)$ by

$$
\bar{E}_{n}(x+1)=-\bar{E}_{n}(x)
$$

for all $x$, and

$$
\bar{E}_{n}(x)=E_{n}(x) \text { for } 0 \leqslant x<1 .
$$

It can be shown that $\bar{E}_{n}(x)$ has continuous derivatives up to the $(n-1)$ st order.

The following is known as Boole's summation formula (see, for example, [9, p. 34]).

LEMMA 1. Let $f(t)$ be a function with $m$ continuous derivatives, defined on the interval $x \leqslant t \leqslant x+\omega$. Then for $0 \leqslant h \leqslant 1$

$$
f(x+h \omega)=\sum_{k=0}^{m-1} \frac{\omega^{k}}{k !} E_{k}(h) \cdot \frac{1}{2}\left(f^{(k)}(x+\omega)+f^{(k)}(x)\right)+R_{m},
$$

where

$$
R_{m}=\frac{1}{2} \omega^{m} \int_{0}^{1} \frac{\bar{E}_{m-1}(h-t)}{(m-1) !} f^{(m)}(x+\omega t) d t .
$$

This summation formula is easy to establish by repeated integration by parts of the above integral. It is remarked in [9, p. 26] that this formula was known to Euler, for polynomial $f$ and without the remainder term. Also note that Lemma 1 turns into Taylor's formula with Lagrange's remainder term if we replace $h$ by $h / \omega$ and let $\omega$ approach zero.

To derive a convenient version of Lemma 1 for the applications we have in mind, we set $\omega=1$ and impose further restrictions on $f$.

LEMMA 2. Let $f$ be a function with $m$ continuous derivatives, defined on $t \geqslant x$. Suppose that $f^{(k)}(t) \rightarrow 0$ as $t \rightarrow \infty$ for all $k=0,1, \ldots, m$. Then for $0 \leqslant h \leqslant 1$

$$
\sum_{v=0}^{\infty}(-1)^{v} f(x+h+v)=\sum_{k=0}^{m-1} \frac{E_{k}(h)}{2 k !} f^{(k)}(x)+R_{m},
$$

where

$$
R_{m}=\frac{1}{2} \int_{0}^{\infty} \frac{\bar{E}_{m-1}(h-t)}{(m-1) !} f^{(m)}(x+t) d t
$$


3. The Remainder for Gregory's Series. The Euler numbers $E_{n}$ may also be defined by the generating function

$$
\frac{2}{e^{t}+e^{-t}}=\sum_{n=0}^{\infty} E_{n} \frac{t^{n}}{n !} .
$$

Comparing (3.1) with (2.1), we see that

$$
E_{n}=2^{n} E_{n}\left(\frac{1}{2}\right)
$$

The phenomenon mentioned in the introduction is entirely explained by the next proposition-if we set $n=500,000$. It is also clear that we will get similar patterns for $n=10^{m} / 2$ with any positive integer $m$.

Proposition 1. For positive integers $n$ and $M$ we have

$$
4 \sum_{k=n}^{\infty} \frac{(-1)^{k}}{2 k+1}=(-1)^{n} \sum_{k=0}^{M} \frac{2 E_{2 k}}{(2 n)^{2 k+1}}+R_{1}(M),
$$

where

$$
\left|R_{1}(M)\right| \leqslant \frac{2\left|E_{2 M}\right|}{(2 n)^{2 M+1}} .
$$

Proof. Apply Lemma 2 with $f(x)=1 / x$; then set $x=n$ and $h=1 / 2$. We get

$$
\sum_{v=0}^{\infty} \frac{(-1)^{v}}{n+v+1 / 2}=\sum_{k=0}^{m-1} \frac{E_{k}(1 / 2)}{2 k !} \frac{(-1)^{k} k !}{n^{k+1}}+R_{m},
$$

with

$$
R_{m}=\frac{1}{2} \int_{0}^{\infty} \frac{\bar{E}_{m-1}(h-t)}{(m-1) !} \frac{(-1)^{m} m !}{(x+t)^{m+1}} d t
$$

We multiply both sides of (3.4) by $2(-1)^{n}$. Then the left-hand side is seen to be identical with the left-hand side of (3.3). After replacing $m$ by $2 M+1$ and taking into account (3.2) and the fact that odd-index Euler numbers vanish, we see that the first terms on the right-hand sides of (3.3) and (3.4) agree. To estimate the error term, we use the following inequality,

$$
\left|E_{2 M}(x)\right| \leqslant 2^{-2 M}\left|E_{2 M}\right| \text { for } 0 \leqslant x \leqslant 1
$$

(see, e.g., [1, p. 805]). Carrying out the integration now leads to the error estimate given in Proposition 1.

4. An Analogue For $\log 2$. Lemma 2 can also be used to derive a result similar to Proposition 1, concerning truncations of the series

$$
\log 2=\sum_{k=1}^{\infty} \frac{(-1)^{k+1}}{k} .
$$

In this case the tangent numbers $T_{n}$ will play the role of the $E_{n}$ in Proposition 1 . It 
follows from the identity

$$
\tan z=\frac{1}{i}\left(\frac{2 e^{2 i z}}{e^{2 i z}+1}-1\right)
$$

together with (1.2) and (2.1) that

$$
T_{n}=(-1)^{n} 2^{n} E_{n}(1)
$$

as in $\left[9\right.$, p. 28]. The $T_{n}$ can be computed using the recurrence relation $T_{0}=1$ and

$$
\sum_{k=0}^{n}\left(\begin{array}{l}
n \\
k
\end{array}\right) 2^{k} T_{n-k}+T_{n}=0 \text { for } n \geqslant 1
$$

Other properties can be found, e.g., in [8] or [9, Ch. 2].

Proposition 2. For positive integers $n$ and $M$ we have

$$
\sum_{k=n+1}^{\infty} \frac{(-1)^{k+1}}{k}=(-1)^{n+1}\left\{\frac{1}{2 n}+\sum_{k=1}^{M} \frac{T_{2 k-1}}{(2 n)^{2 k}}\right\}+R_{2}(M),
$$

where

$$
\left|R_{2}(M)\right| \leqslant \frac{\left|E_{2 M}\right|}{(2 n)^{2 M+1}}
$$

Proof. We proceed as in the proof of Proposition 1. Here we take $x=n$ and $h=1$. Using (4.2) and the fact that $T_{0}=1$ and $T_{2 k}=0$ for $k \geqslant 1$, we get the summation on the right-hand side of (4.3). The remainder term is estimated as in the proof of Proposition 1.

Using Proposition 2 with $n=10^{m} / 2$ one again gets many more correct digits of $\log 2$ than is suggested by the error term of the Taylor series.

5. Generalizations. Proposition 1 and 2 can be extended easily in two different directions.

i). The well-known infinite series (see, e.g., $[1$, p. 807])

$$
\sum_{k=0}^{\infty} \frac{(-1)^{k}}{(2 k+1)^{2 n+1}}=\frac{\left|E_{2 n}\right|}{2^{2 n+2}(2 n) !} \pi^{2 n+1} \quad(n=0,1, \ldots),
$$

and

$$
\begin{aligned}
\sum_{k=1}^{\infty} \frac{(-1)^{k-1}}{k^{2 n}} & =\left(1-2^{1-2 n}\right) \zeta(2 n) \\
& =\left(2^{2 n-1}-1\right) \frac{\left|B_{2 n}\right|}{(2 n) !} \pi^{2 n} \quad(n=1,2, \ldots)
\end{aligned}
$$

can be considered as extensions of Gregory's series and of (4.1). These series admit exact analogues to Propositions 1 and 2; one only has to replace $f(x)=1 / x$ by $f(x)=x^{-(2 n+1)}$, respectively $x^{-(2 n)}$, in the proofs. 
We note that the Euler-MacLaurin summation formula leads to similar results for

$$
\sum_{k=1}^{\infty} k^{-2 n}=\frac{\left|B_{2 n}\right| 2^{2 n-1}}{(2 n) !} \pi^{2 n},
$$

where multiples of the Bernoulli numbers $B_{2 n}$ take the place of the $E_{n}$ and $T_{n}$ in Propositions 1 and 2.

ii). A generalization of the Euler-MacLaurin and Boole summation formulas was derived by Berndt [3]. This can be applied to character analogues of the series (5.1)-(5.3). The roles of the $E_{n}$ and $T_{n}$ in Proposition 1 and 2 are then played by generalized Bernoulli numbers or by related numbers.

6. Additional Comments. The phenomenon observed in the introduction results from taking $N$ to be a power of ten; taking $N=2 \cdot 10^{m}$ also leads to "clean" expressions. References [1], [5], [6], and [9] include the basic material on Bernoulli and Euler numbers, while [8] deals extensively with their calculation, and [2] describes an entertaining analogue of Pascal's triangle. Much on the calculation of pi and related matters may be found in [4]. Euler summation is treated in [5], [6], and [9], while Boole summation is treated in [9]. Related material on the computation and acceleration of alternating series is given in [7].

Added in Proof. A version of the phenomeon was observed by M. R. Powell and various explanations were offered (see The Mathematical Gazette, 66 (1982) 220-221, and 67(1983) 171-188).

\section{REFERENCES}

1. M. Abramowitz and I. Stegun, Handbook of Mathematical Functions, Dover, N.Y., 1964.

2. M. D. Atkinson, How to compute the series expansions of $\sec x$ and $\tan x$, Amer. Math. Monthly, 93 (1986) 387-388.

3. B. C. Berndt, Character analogues of the Poisson and Euler-MacLaurin summation formulas with applications, J. Number Theory, 7 (1975 413-445.

4. J. M. Borwein and P. B. Borwein, Pi and the AGM - A Study in Analytic Number Theory and Computational Complexity, Wiley, N.Y., 1987.

5. T. J. I'a Bromwich, An Introduction to the Theory of Infinite Series, 2nd ed., MacMillan, London, 1926.

6. R. L. Graham, D. E. Knuth, and O. Patashnik, Concrete Mathematics, Addison Wesley, Reading, Mass., 1989.

7. R. Johnsonbaugh, Summing an alternating series, this MoNTHLY, 86 (1979) 637-648.

8. D. E. Knuth and T. J. Buckholtz, Computation of Tangent, Euler, and Bernoulli numbers, Math. Comput., 21 (1967) 663-688.

9. N. Nörlund, Vorlesungen über Differenzenrechnung, Springer-Verlag, Berlin, 1924.

10. R. D. North, personal communications, 1988.

11. N. J. A. Sloane, A Handbook of Integer Sequences, Academic Press, New York, 1973. 